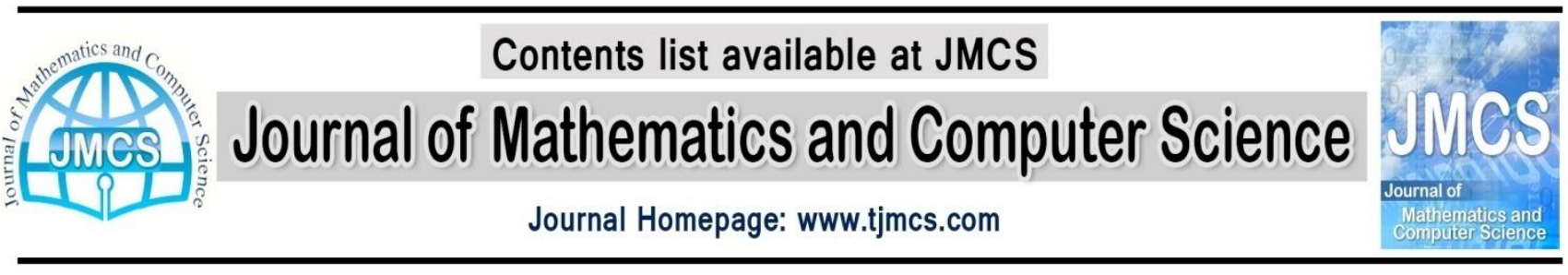

\title{
On Estimation of Parameters of Lagrangian Katz Distribution
}

\author{
N. Abbasi ${ }^{1, *}$, Y. Najmi ${ }^{1}$ \\ ${ }^{1}$ Department of Statistics, Payame Noor University, Tehran, Iran \\ "n_abbasi@pnu.ac.ir \\ Article history: \\ Received July 2014 \\ Accepted October 2014 \\ Available online November 2014
}

\section{Abstract}

We have provided a generalized form of improved estimators by promoting usual estimators included in Lagrangian Katz distribution under a weighted squared error loss. As there are several forms derives of this distribution, the results can be employed for other distributions of this family, as well.

Keywords: Improved estimator, Lagrangian Katz, Risk function, weighted squared error loss function.

\section{Introduction}

Johnson (1987) presented simultaneous estimators of sample size in binomial distributions similar to the method of Hwang (1982) and Ghost and et al. (1983). Later, Grevstad (2011) produced the simultaneous estimators of negative binomial distributions parameter $\mathrm{s}$ when the success probabilities are known.

Many models have applications in industry and medicine is done by a discrete distribution. (See: Jamkhaneh, and et al (2010)) In this paper we take more general consideration to present an estimator of Lagrangian Katz that dominates the usual estimators. It is obvious that the results provided by this paper could be generalized to other members of Katz subcategory. It is known to characterize the Poisson, negative binomial and binomial distributions. Gathy and Lefèvre (2010) used the Lagrangian Katz family of distributions as a claim frequency model.

Lagrangian Katz distribution with parameters $\alpha>0$ and $\beta<1$ has the following probability mass function.

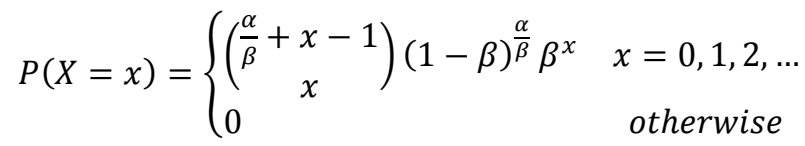

We rewrite the distribution by changing variables $(A=\alpha / \beta)$, as follows

$P(X=x)=\left(\begin{array}{c}A+x-1 \\ x\end{array}\right)(1-\beta)^{A} \beta^{x}$ 
In this case, the expected distribution is equal to $E(X)=A \beta(1-\beta)^{-1}$

We can write unbiased estimator as $E(X(1-\beta) / \beta)=A$ when $\beta$ is known, hence $X(1-\beta) / \beta$ is unbiased estimator for $A$. To find dominate estimator of $X(1-\beta) / \beta$ for $A$ is purpose, as

$\frac{X(1-\beta)}{\beta}=\left(\frac{X_{1}\left(1-\beta_{1}\right)}{\beta_{1}}, \ldots, \frac{X_{m}\left(1-\beta_{m}\right)}{\beta_{m}}\right)^{t}$,

$A=\left(A_{1}, \ldots, A_{m}\right)^{t}$.

Let $X=\left(X_{1}, \ldots, X_{m}\right)^{t}, X_{i} ; 1 \leq i \leq m$, be independent random variables having Katz distribution with $\beta_{i}$ and $\alpha_{i}$ parameters, for a known $\beta_{i}$ and an unknown $\alpha_{i}$. We construct estimators which dominate $\mathrm{X}(1-\beta) / \beta$ for estimating $\mathrm{A}$ with following weighted squared error loss function:

$L(\delta(X), A)=\sum_{i=1}^{m} k_{i}^{2}\left(\delta_{i}(X)-A_{i}\right)^{2}$.

\section{Dominance results}

Consider the dominate estimator of $\mathrm{X}(1-\beta) / \beta$ has the following form:

$\frac{X(1-\beta)}{\beta}+g(X)$

To obtain dominates estimator on $X(1-\beta) / \beta$, we use the difference in risk between $X(1-\beta) / \beta$ and $X(1-\beta) / \beta+g(X)$, for this purpose, the following lemma will prove to have been in this lemma defines $\Delta_{i} g(X)=g(X)-g\left(X-e_{i}\right)$ when $g$ is a multivariate function, where $\mathrm{e}_{\mathrm{i}}$ the unit vector is whose $i$ th component is 1 .

Lemma 2.1. Let $X$ has Lagrangian Katz distribution in (2), then

$E\left[\left(\frac{X(1-\beta)}{\beta}\right) g(X)\right]=\frac{1}{\beta} E(X \Delta g(X))$

Proof: To prove the lemma that $E(X \Delta g(X))$ the obtained traces are as:

$$
\begin{aligned}
E(A g(X)) & =\sum_{x} A \frac{(A+x-1) !}{x !(A-1) !}(1-\beta)^{A} \beta^{x} g(x) \\
& =\sum_{x}((A+x)-x) \frac{(A+x-1) !}{x !(A-1) !}(1-\beta)^{A} \beta^{x} g(x) \\
& =\sum_{x}(x+1) \frac{(A+x-1) !}{(x+1) !(A-1) !}(1-\beta)^{A} \beta^{x} g(x)-E(X g(X)) \\
& =\frac{1}{\beta} \sum_{x}(x+1) \frac{(A+x-1) !}{x !(A-1) !}(1-\beta)^{A} \beta^{x+1} g(x)-E(X g(X))
\end{aligned}
$$

by changing variables $y=x+1$, 


$$
E(A g(X))=\frac{1}{\beta} E(X g(X-1))-E(X g(X))
$$

Now we have:

$$
\begin{aligned}
E\left[\left(\frac{X(1-\beta)}{\beta}-A\right) g(X)\right] & =\frac{(1-\beta)}{\beta} E(X g(X))-E(A g(X)) \\
& =\frac{(1-\beta)}{\beta} E(X g(X))-E(X g(X))-\frac{1}{\beta} E(X g(X-1)) \\
& =\frac{1}{\beta} E(X g(X))-\frac{1}{\beta} E(X g(X-1)) \\
& =\frac{1}{\beta} E(X \Delta g(X))
\end{aligned}
$$

Now obtain difference risk for estimator $X(1-\beta) / \beta+g(X)$ and $X(1-\beta) / \beta$, we proved:

$$
\begin{aligned}
& R\left(\frac{X(1-\beta)}{\beta}, A\right)-R\left(\frac{X(1-\beta)}{\beta}+g(X), A\right)<0 . \\
& R\left(\frac{X(1-\beta)}{\beta}, \mathrm{A}\right)-R\left(\frac{X(1-\beta)}{\beta}+\mathrm{g}(\mathrm{X}), \mathrm{A}\right)=\mathrm{E}\left(\sum_{\mathrm{i}=1}^{\mathrm{m}} \mathrm{K}_{\mathrm{i}}^{2}\left(\frac{\mathrm{X}_{\mathrm{i}}\left(1-\beta_{\mathrm{i}}\right)}{\beta_{\mathrm{i}}}-\mathrm{A}_{\mathrm{i}}\right)^{2}\right) \\
&-\mathrm{E}\left(\sum_{\mathrm{i}=1}^{\mathrm{m}} \mathrm{K}_{\mathrm{i}}^{2}\left(\frac{\mathrm{X}_{\mathrm{i}}\left(1-\beta_{\mathrm{i}}\right)}{\beta_{\mathrm{i}}}+\mathrm{g}(\mathrm{X})-\mathrm{A}_{\mathrm{i}}\right)^{2}\right) \\
&= \mathrm{E}\left(\sum _ { \mathrm { i } = 1 } ^ { \mathrm { m } } \mathrm { K } _ { \mathrm { i } } ^ { 2 } \left(\frac{\mathrm{X}_{\mathrm{i}}\left(1-\beta_{\mathrm{i}}\right)}{\left.\left.\beta_{\mathrm{i}}-\mathrm{A}_{\mathrm{i}}\right)^{2}\right)}\right.\right. \\
&-\mathrm{E}\left(\sum_{\mathrm{i}=1}^{\mathrm{m}} \mathrm{K}_{\mathrm{i}}^{2}\left(\left(\frac{\mathrm{X}_{\mathrm{i}}\left(1-\beta_{\mathrm{i}}\right)}{\beta_{\mathrm{i}}}-\mathrm{A}_{\mathrm{i}}\right)^{2}+\mathrm{g}(\mathrm{X})^{2}\right)\right) \\
&+ \mathrm{E}\left(\sum_{\mathrm{i}=1}^{\mathrm{m}} \mathrm{K}_{\mathrm{i}}^{2}(2 \mathrm{X})\left(\frac{\mathrm{X}_{\mathrm{i}}\left(1-\beta_{\mathrm{i}}\right)}{\beta_{\mathrm{i}}}-\mathrm{A}_{\mathrm{i}}\right)\right.
\end{aligned}
$$

Using Lemma (1) we have:

$$
R\left(\frac{X(1-\beta)}{\beta}, \mathrm{A}\right)-R\left(\frac{X(1-\beta)}{\beta}+\mathrm{g}(\mathrm{X}), \mathrm{A}\right)=-\mathrm{E}\left(\sum_{\mathrm{i}=1}^{\mathrm{m}}\left(2 \mathrm{~K}_{\mathrm{i}}^{2} \frac{1}{\beta_{\mathrm{i}}} \mathrm{X}_{\mathrm{i}} \Delta \mathrm{g}_{\mathrm{i}}(\mathrm{X})+\mathrm{K}_{\mathrm{i}}^{2} \mathrm{~g}_{\mathrm{i}}(\mathrm{X})^{2}\right)\right) \geq 0
$$

and hence

$$
\mathrm{E}\left(\sum_{\mathrm{i}=1}^{\mathrm{m}}\left(\mathrm{K}_{\mathrm{i}}^{2} \frac{1}{\beta_{\mathrm{i}}} \mathrm{X}_{\mathrm{i}} \Delta \mathrm{g}_{\mathrm{i}}(\mathrm{X})+\frac{1}{2} \mathrm{~K}_{\mathrm{i}}^{2} \mathrm{~g}_{\mathrm{i}}(\mathrm{X})^{2}\right)\right) \leq 0
$$

Now with comparing obtained inequality with the difference inequality

$\sum\left\{v_{i}\left(X_{i}\right) \Delta_{i} \Psi_{i}(X)+w_{i}(X) \Psi_{i}^{2}(X)\right\} \leq 0$

The results are obtained 
$\Psi_{i}(X)=k_{i} g_{i}(X), \quad w_{i}(X)=\frac{1}{2} \quad v_{i}(X)=\mathrm{K}_{\mathrm{i}} \frac{1}{\beta_{\mathrm{i}}} \mathrm{X}_{\mathrm{i}}$

To get dominate estimator on $\mathrm{X}(1-\beta) / \beta$, we change this estimator at first to a fixed point and then to a database points set.

We act follow the same method of Johnson (1987) for this reason, we define:

$h_{i}(a) \equiv \sum_{j=1}^{a} v_{i}^{-1}(j)=\frac{\beta_{i}}{k_{i}} \sum_{j=1}^{a} \frac{1}{j}$

Also we show the positive part of function $\mathrm{c}(\mathrm{X})$ with $\{\mathrm{c}(\mathrm{X})\}_{+}$.and we define $\#\{A\}$ the cardinality of set A. moreover define $\mathrm{N}(\mathrm{X})=\#\left\{\mathrm{i}: \mathrm{X}_{\mathrm{i}}>\lambda_{\mathrm{i}}\right\}$. $\lambda_{\mathrm{j}}$ is an integer which is attained according to previous data about $A_{i}$.

\section{The ways to find dominate estimator}

To find dominate estimator, we rebuild and change usual estimator. One the of ways is to shrink the usual estimator toward a fixed point, the other way shift this estimator toward a data-based point.

\subsection{Dominate estimator related to a fixed point}

Theorem 3.1. Let $\mathrm{X}=\left(\mathrm{X}_{1}, \ldots, \mathrm{X}_{\mathrm{m}}\right)^{\mathrm{t}}$ be a vector of impendent random variables whose $i$ th component has a Lagrangian Katz distribution with $\beta_{i}$ And $\alpha_{i}$ parameters, that $\beta_{i}$ is known and $\alpha_{i}\left(\right.$ also $\left.A_{i}\right)$ unknown for all $\mathrm{i}$, as $i=1, \ldots, m$ and $m \geq 3$. Then $\frac{X(1-\beta)}{\beta}-\frac{\left[(N(X)-2)_{+}\right]}{D} B(X)$ dominates $\frac{X(1-\beta)}{\beta}$. Under loss function (5), as

$B \equiv B(X) \equiv\left(\frac{H_{1}\left(X_{1}\right)}{K_{1}}, \ldots, \frac{H_{m}\left(X_{m}\right)}{K_{m}}\right)^{t}$

$D \equiv D(X) \equiv \sum_{i=1}^{m} d_{i}\left(X_{i}\right)$

(i) Let

$H_{i}\left(X_{i}\right)=h_{i}\left(X_{i}\right)-h_{i}\left(X_{(n)}\right)$,

$d_{i}\left(X_{i}\right)= \begin{cases}H_{i}^{2}\left(X_{i}\right)+\frac{\beta_{i}}{k_{i}}\left[\frac{3}{2} h_{i}\left(X_{(n)}\right)-\frac{\beta_{i}}{k_{i}}\right]_{+} & X_{i}<X_{(n)} \\ H_{i}\left(X_{i}\right) H_{i}\left(X_{i}+e_{i}\right) & X_{i} \geq X_{(n)}\end{cases}$

(ii) For fixed nonnegative $\varphi_{\mathrm{i}}$ :

$H_{i}\left(X_{i}\right)= \begin{cases}-\varphi_{\mathrm{i}} & X_{i}<X_{(n)} \\ h_{i}\left(X_{i}\right)-h_{i}\left(X_{(n)}\right) & X_{i} \geq X_{(n)}\end{cases}$

$d_{i}\left(X_{i}\right)= \begin{cases}\varphi_{i}^{2} & X_{i}<X_{(n)} \\ H_{i}\left(X_{i}\right) H_{i}\left(X_{i}+e_{i}\right) & X_{i} \geq X_{(n)}\end{cases}$

Proof. See Ghosh and et al. (1983) and Hwang (1982) for details.

\subsection{Dominate estimator related to a data-based point}


To get dominate estimator in this state, we set $X_{(n)}$ as $\mathrm{n}$ of order statistic $X$. The next theory will describe dominate estimator on usual estimator by changing each elements of $X_{(n)}$.

Theorem 3.2. Let $X=\left(X_{1}, \ldots, X_{m}\right)^{t}$ be a vector of independent random variables whose ith component has a Lagrangian Katz distribution with $\beta_{i}$ And $\alpha_{i}$ parameters, that $\beta_{i}$ is known and $\alpha_{i}\left(\right.$ also $\left.A_{i}\right)$ unknown for all $\mathrm{i}$, as $i=1, \ldots, m$ and $m \geq 4$ and $1 \leq \mathrm{n} \leq \mathrm{m}-3$. Then $\frac{X(1-\beta)}{\beta}-$ $\frac{\left[(N(X)-2)_{+}\right]}{D} B(X) \quad$ dominates $\frac{X(1-\beta)}{\beta}$. As

$B \equiv B(X)=\left(\frac{H_{1}\left(X_{1}\right)}{K_{1}}, \ldots, \frac{H_{m}\left(X_{m}\right)}{K_{m}}\right)^{t}$

$D \equiv D(X) \equiv \sum_{i=1}^{m} d_{i}\left(X_{i}\right)$

(i) Let

$$
\begin{aligned}
& H_{i}\left(X_{i}\right)=h_{i}\left(X_{i}\right)-h_{i}\left(X_{(n)}\right) \\
& d_{i}\left(X_{i}\right)= \begin{cases}H_{i}^{2}\left(X_{i}\right)+\frac{\beta_{i}}{k_{i}}\left[\frac{3}{2} h_{i}\left(X_{(n)}\right)-\frac{\beta_{i}}{k_{i}}\right]_{+} & X_{i}<X_{(n)} \\
H_{i}\left(X_{i}\right) H_{i}\left(X_{i}+e_{i}\right) & X_{i} \geq X_{(n)}\end{cases}
\end{aligned}
$$

(ii) For fixed nonnegative $\varphi_{\mathrm{i}}$ :

$$
\begin{aligned}
& H_{i}\left(X_{i}\right)= \begin{cases}-\varphi_{\mathrm{i}} & X_{i}<X_{(n)} \\
h_{i}\left(X_{i}\right)-h_{i}\left(X_{(n)}\right) & X_{i} \geq X_{(n)}\end{cases} \\
& d_{i}\left(X_{i}\right)= \begin{cases}\varphi_{i}^{2} & X_{i}<X_{(n)} \\
H_{i}\left(X_{i}\right) H_{i}\left(X_{i}+e_{i}\right) & X_{i} \geq X_{(n)}\end{cases}
\end{aligned}
$$

Proof. Similar proof of theorem 3.1.

\section{Results}

Comparing dominate estimator which obtained of Katz distribution in this paper and obtained estimators by Johnson (1987) for binomial distribution and too Grevstad (2011) corollary in negative binomial distribution, we can reach to this result that if the variable which change Lagrangian Katz distribution to binomial distribution and negative binomial distribution take to dominate estimator of Lagrangian Katz distribution, we can attain to the result of Johnson and Grevstad achieved. We show this as follows.

In Katz distribution $v_{i}(X)=\mathrm{K}_{\mathrm{i}} \frac{1}{\beta_{\mathrm{i}}} \mathrm{X}_{\mathrm{i}}$ (as for relation (7)) respecting the Katz distribution changes to binomial distribution with accept of this proposes in below, we have

$$
\frac{\alpha}{\beta}=-n, p=\beta(\beta-1)^{-1}, \beta<0
$$

So, $\beta=\frac{\mathrm{p}}{\mathrm{p}-1}$ and as regards $\mathrm{v}(\mathrm{X})>0$ then

$$
v(X)=k \frac{1}{\beta} X=k \frac{1}{\frac{p}{1-p}} X=\frac{k(1-p)}{p} X
$$


That similarity results in Johnson (1987). Also For negative binomial distribution, Lagrangian Katz distribution will change to negative binomial distribution regarding the following qualification:

$$
\frac{\alpha}{\beta}=r, \quad 0<1-\beta=p<1
$$

Hence $\beta=1-p$ then

$\mathrm{v}(\mathrm{X})=\mathrm{k} \frac{1}{\beta} \mathrm{X}=\mathrm{k} \frac{1}{1-\mathrm{p}} \mathrm{X}=\frac{\mathrm{k}}{1-\mathrm{p}} \mathrm{X}$

This is likeness $\mathrm{v}(\mathrm{X})$ in Grevstad (2011).

When $\beta \rightarrow 0$ and $\frac{\alpha}{\beta}=\mathrm{n}$, Lagrangian Katz distribution tends to Poisson distribution, we consider $k$ such that $\frac{\mathrm{k}}{\beta}=1$ so

$\mathrm{v}(\mathrm{X})=\mathrm{k} \frac{1}{\beta} \mathrm{X}=\mathrm{X}$

That v (X) obtained by Ghosh et al. (1983) for the Poisson distribution.

\section{References}

[1] E. B. Jamkhaneh, Sadeghpour- Gildeh, Gh. Yari, "Acceptance Single Sampling Plan By Using of Poisson Distribution", Journal of Mathematics and Computer Science, Vol. 1 (2010), No. 1. pp. 6-13.

[2] J. T. Hwang, "Improving upon standard estimators in discrete exponential families with application to Poisson and negative binomial cases", Annals of Statistics, 10 (33) (1982), pp 857867.

[3] M. Gathy, C. Lafevre, "On the Lagrangian Katz family of distributions as a claim frequency model, Insurance", Mathematics and Economics, Vol 47 (2010), Issue 1, 79-83.

[4] M. Ghosh, J. T. Hwang, K. W. Tusi, "Construction of improved estimators in multiparameter estimation for discrete exponential families", Annals of Statistics, 11(2) (1983), pp 351-367.

[5] N. Grevstad, "Simultaneous estimation of negative binomial dispersion parameters, Statistics and Probability Letters", 81 (2011), pp 1751-1755.

[6] R. W. Johnson, "Simultaneous estimation of binomial N's, Sankhya", The Indian Journal of Statistics, Vol. 49 (1987), Series A, Part 2, pp 264-266. 